\title{
Electronic excitations of benzene from the equations of motion method $^{*}$
}

\author{
John B. Rose ${ }^{\dagger}$, Tai-Ichi Shibuya ${ }^{\ddagger}$, and Vincent McKoy \\ Arthur Amos Noyes Laboratory of Chemical Physics, ${ }^{\S}$ California Institute of Technology, Pasadena, California 91109 \\ (Received 22 March 1973)
}

\begin{abstract}
We have used the equations of motion method to calculate the excitation energies and intensities of several transitions in benzene. The ordering of the singlet and triplet states of $B_{2 u}, B_{1 u}$, and $E_{1 u}$ symmetry agrees with experiment and the error in the calculated frequencies ranges from $3 \%$ to $25 \%$. This error range is reasonable considering the relatively small basis set used. The most extensive calculation included 10 hole and 28 particle states and shows the effect of changes in the sigma core for each transition. The calculated transition moment of 1.74 a.u. for the ${ }^{1} A_{1 \mathrm{~g}}{ }^{1} E_{1 \mathrm{u}}$ transition agrees well with the experimental value of $1.61 \mathrm{a} . \mathrm{u}$.
\end{abstract}

\section{INTRODUCTION}

In recent papers ${ }^{1,2}$ we have developed the equationsof-motion method as an approach specifically designed to study the spectroscopic and response properties of atoms and molecules. In these methods one directly obtains the transition amplitudes of physical interest in a given process, e.g., in photon absorption and in electron-molecule scattering, thereby avoiding many of the difficulties involved in obtaining highly accurate wavefunctions. We have used this method to study the excitation frequencies and transition moments of several molecules, e.g., $\mathrm{N}_{2}, \mathrm{CO}, \mathrm{C}_{2} \mathrm{H}_{4},{ }^{2} \mathrm{H}_{2} \mathrm{CO},{ }^{3}$ and $\mathrm{CO}_{2}{ }^{4}$ and the Born differential cross sections for the scattering of electrons by $\mathrm{CO}_{2} \cdot{ }^{4}$ The results generally agree well with experiment. These results indicate that the method is practical and is capable of including the major correlation effects on the physical observables in a relatively small matrix problem, even for quite large basis sets.

In this paper we present the results of calculations on the excited states of benzene as an application of the method to a larger polyatomic molecule. These calculations were done with a relatively limited basis set and include results both with and without sigma-pi coupling. The inclusion of two particle-two hole $(2 p-2 h)$ excitations also shows the effect of changes in the sigma core on each excitation. Sigma-pi coupling affects mainly the ${ }^{1} A_{1 e}-{ }^{1} E_{1 u}$ transition where it lowers the oscillator strength by about $40 \%$. The ordering of the singlet and triplet states of $B_{2 u}, B_{1 u}$, and $E_{1 u}$ symmetry agrees with experiment and the error in the calculated frequencies ranges from $3 \%$ to $25 \%$. This is primarily due to the relatively small basis set used in the calculation. We also obtain a ${ }^{1} A_{2 u}\left(\sigma-\pi^{*}\right)$ state at $9.3 \mathrm{eV}$ with an oscillator strength of about 0.03 .

In the next section we give a few equations outlining the equations-of-motion method. Section III gives the results of the calculations on benzene.

\section{THEORY}

Here we review some essential results of the equations-of-motion method. The operator $O_{\lambda}^{+}$which generates an excited state $|\lambda\rangle$ from the ground state is exactly a solution of the equation, ${ }^{5}$

$$
\left\langle 0\left|\left[\delta O_{\lambda}, H, O_{\lambda}^{+}\right]\right| 0\right\rangle=\omega_{\lambda}\left\langle 0\left|\left[\delta O_{\lambda}, O_{\lambda}^{+}\right]\right| 0\right\rangle,
$$

for all variations $\delta O_{\lambda}$. The double commutator is defined as

$$
2[A, H, B]=[A,[H, B]]+[[A, H], B]
$$

and $\omega_{\lambda}$ is the excitation frequency. The amplitudes of $O_{\lambda}^{*}$ are elements of the transition density for the excitation $|0\rangle-|\lambda\rangle$. With $O_{\lambda}^{+}$restricted to the single particlehole $(1 p-1 h)$ form, Eq. (1) reduces to the matrix equation for the amplitudes $\left\{Y_{m y}\right\}$ and $\left\{Z_{m}\right\}$,

$$
\left[\begin{array}{cc}
\mathrm{A} & \mathrm{B} \\
-\mathrm{B}^{*} & -\mathrm{A}^{*}
\end{array}\right]\left[\begin{array}{l}
Y(\lambda) \\
Z(\lambda)
\end{array}\right]=\omega_{\lambda}\left[\begin{array}{ll}
\mathrm{D} & O \\
O & \mathrm{D}
\end{array}\right]\left[\begin{array}{l}
Y(\lambda) \\
Z(\lambda)
\end{array}\right] .
$$

The elements of A, B, and D are given elsewhere. ${ }^{1}$

We have also shown that the equation of motion for $O_{\lambda}^{+}$ including two-particle-two-hole $(2 p-2 h)$ components can be put in the form of Eq. (3). ${ }^{6}$ The renormalized matrices now depend on the frequencies, $\omega_{\lambda}$, and are hence energy dependent. The amplitudes of Eq. (3) determine the transition moment $M_{0 \lambda}$ by the simple relation,

$$
M_{o \lambda}=\sqrt{2} \sum_{m \gamma}\left[Y_{m \gamma}^{*}(\lambda) M_{m_{\gamma}}+Z_{m \gamma}^{*}(\lambda) M_{m_{\gamma}}\right],
$$

where $M_{m \gamma}$ is a particle-hole matrix element of $M$. The oscillator strength, $f_{o \lambda}$, is then

$$
f_{o \lambda}=\frac{2}{3} G \omega_{\lambda} M_{o \lambda}^{2}
$$

where $G$ is the degeneracy factor. The major effect of the $(2 p-2 h)$ components of $O_{\lambda}^{+}$on the transition amplitude can be included by renormalizing the $(1 p-1 h)$ amplitudes.

\section{RESULTS AND DISCUSSION}

The first step in our calculation is to carry out an SCF calculation on the ground state of benzene to obtain a suitable particle-hole basis (unoccupied-occupied orbitals). The ground state electronic configuration is

$$
\begin{aligned}
& 1 a_{1 g}^{2} 1 e_{1 u}^{4} 1 e_{2 \xi}^{4} 1 b_{1 u}^{2} 2 a_{1 g}^{2} 2 e_{1 u}^{4} 2 e_{2 g}^{4} \\
& \quad \times 3 a_{1 \xi}^{2} 2 b_{1 u}^{2} 1 b_{2 u}^{2} 3 e_{1 u}^{4} 1 a_{2 u}^{2} 3 e_{2 \xi}^{4} 1 e_{1 \xi}^{4} .
\end{aligned}
$$

In this calculation we used a $[3 s 2 p / 1 s]$ Gaussian basis contracted from a primitive $(7 s 3 p / 3 s)$ basis. Table I lists the eigenvalues for the 21 occupied orbitals and the first 28 virtual (particle) states. The total energy of this SCF calculation is -230.2184 a. $\mathrm{u}$. 
For the first set of calculations all $1 p-1 h$ and $2 p-2 h$ pairs that can be generated from the hole states $3 a_{1 s}$, $2 b_{u u}, 1 b_{u u}, 3 e_{k u}, 1 a_{2 u}, 3 e_{2 \xi}$, and $1 e_{1 g}$ and all the particle states in Table I are used to solve the equations of motion for transitions to the ${ }^{1} B_{3 u},{ }^{1} B_{1 u},{ }^{1} E_{1 u},{ }^{1} E_{2 z}$ states and their corresponding triplet states. This calculation then includes 10 hole states and 28 particle states. The truncation of 60 molecular orbitals should still allow the major effects described by this basis to display themselves. The number of $1 p-1 h$ pairs included are 34,21 , 24 , and 34 for the $E_{1 u}, B_{2 u}, B_{1 u}$, and $E_{2 g}$ states, respectively. Table II shows the results of this calculation in both the $1 p-1 h$ approximation and the $(1 p-1 h)+(2 p-2 h)$ approximation. These results include, among other effects, the effects of sigma-pi coupling and relaxation of the sigma and pi systems. We will discuss these results in the next paragraph. In Table I we also give the excitation frequencies for these transitions in the $\pi$ electron approximation which therefore include particlehole pairs from the $1 a_{2 u}$ and $1 e_{1 g}$ hole states and $1 b_{2 g}$, $2 a_{2 u}, 2 e_{1 g}, 2 e_{2 u}$, and $2 b_{2 z}$ particle states. In the (1p$1 h)+(2 p-2 h)$ approximation these excitation frequencies also include the effect of relaxation of the $\pi$ system in the excitation process.

The excitation energies for the complete calculation, i.e., including sigma and pi particle-hole pairs in the $(1 p-1 h)+(2 p-2 h)$ approximation compare well with experiment. The ordering of the states is correct and the error range for the frequencies is reasonable considering the relatively small basis set, i. e., $[3 s 2 p / 1 s]$. The differences between the calculated and observed excitation frequencies becomes larger for the higher states where double and multiple excitations and a more flexible basis are expected to be more important. The results in the $\pi$ approximation agree well with those including excitations out of the sigma and pi systems except for the ${ }^{3} B_{1 u}$ and ${ }^{1} E_{1 u}$ states. The increase of $1.5 \mathrm{eV}$ for the ${ }^{3} B_{1 u}$ excitation energy in going from the $(1 p-1 h) \pi$

TABLE I. SCF orbital eigenvalues for $\mathrm{C}_{6} \mathrm{H}_{6} \cdot{ }^{2}$

\begin{tabular}{|c|c|c|c|}
\hline MO & $\epsilon_{i}^{b}$ & MO & $\epsilon_{i}$ \\
\hline $\begin{array}{l}1 a_{1 z} \\
1 e_{1 u} \\
1 e_{2 z} \\
1 b_{1 u} \\
2 a_{1 g} \\
2 e_{1 u} \\
2 e_{2 g} \\
3 a_{1 g} \\
1 b_{2 u} \\
2 b_{2 u} \\
3 e_{1 u} \\
1 a_{2 u} \\
3 e_{2 g} \\
1 e_{1 g} \\
1 e_{2 u} \\
1 b_{2 g}\end{array}$ & $\begin{array}{r}-11.3218 \\
-11.3224 \\
-11.3215 \\
-11.3214 \\
-1.1945 \\
-1.0625 \\
-0.8704 \\
-0.7470 \\
-0.6768 \\
-0.6731 \\
-0.6276 \\
-0.5561 \\
-0.5336 \\
-0.3889 \\
0.0984 \\
0.3187\end{array}$ & $\begin{array}{l}4 a_{1 \xi} \\
4 e_{1 u} \\
4 e_{2 g} \\
3 b_{1 u} \\
5 e_{2 g} \\
5 e_{1 u} \\
1 a_{2 g} \\
2 b_{2 u} \\
2 a_{2 u} \\
5 a_{1 \xi} \\
4 b_{1 u} \\
6 e_{2 g} \\
2 e_{1 \xi} \\
2 e_{2 u} \\
6 e_{1 u} \\
2 b_{2 g} \\
2 a_{2 g}\end{array}$ & $\begin{array}{l}0.3327 \\
0.4187 \\
0.5149 \\
0.5242 \\
0.6441 \\
0.6447 \\
0.7572 \\
0.9238 \\
0.9897 \\
0.9988 \\
0.9996 \\
1.0574 \\
1.0859 \\
1.1305 \\
1.1735 \\
1.1995 \\
1.4051\end{array}$ \\
\hline
\end{tabular}

In a $[3 s 2 p / 1 s]$ basis. Total energy is -230.2184 a.u.

In atomic units.
TABLE II. Excitation energies of benzene. a

\begin{tabular}{lccccc}
\hline & \multicolumn{4}{c}{$\Sigma-\Pi^{\mathrm{d}}$} \\
\hline State & $(1 p-1 h)$ & $(1 p-1 h)+(2 p-2 h)$ & $(1 p-1 h)$ & $(1 p-1 h)+(2 p-2 h)$ & Obs \\
${ }^{1} B_{2 u}$ & 7.7 & 5.7 & 8.5 & 5.5 & 4.9 \\
${ }^{1} B_{1 u}$ & 8.4 & 7.8 & 8.7 & 7.4 & 6.1 \\
${ }^{1} E_{2 u}$ & 10.7 & 9.5 & 10.5 & 8.3 & 6.9 \\
${ }^{1} \Sigma_{2 v}$ & 12.9 & 11.3 & 12.8 & 10.9 & $\cdots .1$ \\
${ }^{3} B_{1 u}$ & 3.0 & 2.7 & 4.5 & 3.8 & 3.7 \\
${ }^{3} E_{1 u}$ & 6.1 & 5.2 & 7.1 & 5.2 & 4.6 \\
${ }^{3} B_{2 u}$ & 7.3 & 7.0 & 8.2 & 6.7 & 5.8 \\
${ }^{3} E_{2 q}$ & 8.5 & 7.9 & 9.4 & 8.1 & $\cdots . \mathrm{f}$ \\
\hline \hline
\end{tabular}

aIn electron volts.

${ }^{b}$ II approximation. See text.

${ }^{C}$ The transition moment for the ${ }^{1} A_{1 g} \rightarrow{ }^{1} E_{1 u}$ transition is 2.09 in this approximation. See text.

dSee text.

${ }^{\text {e }}$ The transition moment for this transition is 1.74 in this approximation. The experimental moment is 1.61 .

isee text.

approximation to the $(1 p-1 h) \sigma-\pi$ theory is presumably because this very low energy state is almost exclusively a $\pi \rightarrow \pi^{*}$ transition and hence $\sigma-\sigma$ correlation is more important than $\sigma-\pi$ coupling. However, we see that changes in the sigma core are important in lowering the excitation energy of the ${ }^{1} E_{14}$ state by $1.2-8.3 \mathrm{eV}$. The calculated excitation energy for the ${ }^{1} E_{2 g}$ state is higher than the estimates obtained by configuration interaction (CI) studies for this transition. ${ }^{7,8}$ We will return to a brief comparison of our results with those of CI studies later but it does seem that either multiple excitations are important in describing this transition or the truncation of our particle states gives too small a $(2 p-2 h)$ contribution.

The only optically allowed transition of those listed in Table II is the ${ }^{1} A_{1 g} \rightarrow{ }^{1} E_{1 u}$ transition. The transition moment in the full calculation including $\sigma-\pi$ coupling is $1.74 \mathrm{a}, \mathrm{u}$. which agrees well with the transition moment of $1.61 \mathrm{a}, \mathrm{u}$. derived from the experimental oscillator strength of $0.88 .^{9}$ In the $\pi$ approximation the transition moment is 2.09 a. u. This change in the transition moment from 2.09 to 1.74 a. u. due to $\sigma-\pi$ coupling leads to a reduction of about $40 \%$ in the oscillator strength. Our results also give a ${ }^{1} A_{2 u}\left(\sigma-\pi^{*}\right)$ state at $9.3 \mathrm{eV}$ with a transition moment of 0.34 a. u.

The available CI calculations ${ }^{7,8}$ on the $\pi-\pi^{*}$ states of benzene do not include as many particle and hole states as the present calculations. For this and other reasons we do not compare our results in any detail with these other results. In general, our results are in slightly better agreement with experiment. The main difference in the predicted excitation energies are for the ${ }^{1} E_{14}$ and ${ }^{1} E_{2 g}$ states. Our excitation energy for the ${ }^{1} A_{1 \xi}-{ }^{1} E_{2 s}$ transition is about $2 \mathrm{eV}$ larger than the other estimates. Part of this difference may be due to the truncation of the particle basis which affects the $2 p-2 h$ contribution for this high singlet state. More extensive calculations could clarify this difference. We do not list experimental values for the ${ }^{1} E_{2 \xi}$ and ${ }^{3} E_{2 g}$ states because no conclusive experimental assignments ${ }^{10,11}$ have been made. Our calculated excitation energy of $8.3 \mathrm{eV}$ for the ${ }^{1} E_{1 u}$ 
state is much closer to the experimental value of $6.9 \mathrm{eV}$ than the previous estimates of $9.4 \mathrm{eV}^{8}$ and $10.6 \mathrm{eV} .^{7}$ This shows the importance of changes in the $\sigma$ core in this excitation. No values for the transition moment for the ${ }^{1} A_{1 g}-{ }^{1} E_{1 u}$ transition are reported in these CI calculations. ${ }^{7,}$

\section{CONCLUSIONS}

We have discussed the results of calculations on the excited states of benzene as an application of the equations of motion method to a larger polyatomic molecule. The ordering of the singlet and triplet states of $B_{2 u}, B_{i u}$, and $E_{1 u}$ symmetry agrees with experiment and the error range of $3 \%$ to $25 \%$ for the calculated frequencies is reasonable considering the limited basis set employed. These calculations show the effects of changes in the sigma core for each transition separately by including particle-hole excitations from the $3 a_{1 g}, 1 b_{2 u}, 2 b_{1 u}$, $3 e_{1 u}$, and $3 e_{2 g}$ core states. The calculations can still be done economically at this level. The calculated transition moment for the ${ }^{1} A_{1 g}-{ }^{1} E_{1 u}$ transition is $1.74 \mathrm{a}$. u. compared to the value of $1.61 \mathrm{a}, \mathrm{u}$. derived from the experimental $f$ value of 0.88 .
*Work supported by the National Science Foundation.

†Present address: CECAM, Batiment 506, Universite de Paris XI, 91-Orsay, France.

Present address: Department of Physics, Lniversity of the Pacific, Stockton, CA.

$\$$ Contribution No. 4663 .

${ }^{\prime}$ T. Shibuya and V. McKoy, 1'hys. Rev. A 2, 2208 (1970).

${ }^{2} \mathrm{~J}$. Rose, T. Shibuya, and V. McKoy, J. Chem. Phys. 58, 74 (1973).

${ }^{3}$ D. L. Yeager and V. McKoy, "Equations of motion method: The excited states of formaldehyde," J. Chem. Phys, (to be published).

${ }^{4} \mathrm{C}$. W. McCurdy and V. McKoy, "Differential cross sections for the scattering of electrons by $\mathrm{CO}_{2}$," J. Chem. Phys. (to be published).

${ }^{5}$ D. J. Rowe, Rev. Mod. Phys. 40, 153 (1968).

${ }^{6}$ T. Shibuya, J. Rose, and V. Mckoy, J. Chem. Phys. 58, 500 (1973).

${ }^{7}$ R. J. Buenker, J. L. Whitten, and J. D. Petke, J. Chem. Phys. 49, 2261 (1968).

${ }^{8}$ S. Peyerimhoff and R. J. Buenker, Theoret. Chim. Acta 19, $1(1970)$.

${ }^{9}$ V. J. Hammond and $W$. C. Price, Trans, Faraday Soc. 51, $605(1955)$.

${ }^{10}$ B. Katz, M. Brith, A. Ron, B. Sharf, and J. Jortner, Chem. Phys. Lett, 2, 189 (1968).

${ }^{11}$ P. R. Monson and W. M. McClain, J. Chem. Phys. 53, 29 (1970). 